\title{
Application of New Formulas for the Spiral Arm Formation to Selected Galaxies with Special Patterns
}

\author{
Hongjun Pan \\ Department of Chemistry, University of North Texas, Denton, Texas, USA \\ Email address: \\ hpan@unt.edu \\ To cite this article: \\ Hongjun Pan. Application of New Formulas for the Spiral Arm Formation to Selected Galaxies with Special Patterns. American Journal of \\ Astronomy and Astrophysics. Vol. 8, No. 3, 2020, pp. 45-60. doi: 10.11648/j.ajaa.20200803.12
}

Received: August 20, 2020; Accepted: August 31, 2020; Published: September 16, 2020

\begin{abstract}
Seven special galaxies with very unusual spiral patterns are precisely simulated and studied by recently proposed ROTASE hypothesis with derived mathematical formulas for the spiral arm formation of the galaxies, fully match the prediction of the model. MCG+00-04-051 shows the broken connection of the spiral arms with the ends of the galactic bar, such broken connection is due to the termination of the X-matter emission by the emission-active black hole located at the center of the galaxy. The weak but still visible arm section in the image clearly proves that the disconnected arms do not rotate ahead of the closest bar ends, they actually rotate behind of the other ends of the bar from which they were generated. The galaxy NGC 4548 has apparent broken connection of spiral arms with bar ends in a low-quality image, such broken connection gives false impression that the arms rotate ahead of the galactic bar ends. However, weak and visible arm in the better-quality image clearly proves that the arms rotate behind the bar ends, same as galaxy MCG+00-04-051. ESO325-28 shows a perfect spiral ring pattern. The galaxy NGC 7098 is made of two identical rings, each ring is made of a half inner ring and a half outer ring, the two rings cross each other twice with chain-link style, this is due to the X-matter emission behavior change with time following Gaussian formula. UGC12646 shows the same pattern as NGC 7098 with two rings crossing each other twice with chain-link style. The pattern image of UGC12646 clearly demonstrates that quality of the spiral arms decreases with their age only, not with their distance to the ends of the bar. NGC 1079 shows the most amazing artwork-like double ring pattern, each spiral arm has three arm crossings: it crosses other arm twice and crosses itself once. Galaxy SDSS J015701.50-001644.4 shows a unique two-spiral arm structure, each arm structure is made of a half inner ring and a whole outer ring; the two outer rings cross each other with chain-link style. The result also shows that the galaxy NGC 4548 has incomplete or destroyed double ring pattern. The possible formation and evolution sequence of the Hoag's object is illustrated with images of real galaxies. Pitch angles of spiral arms can be easily calculated after successful simulation of spiral patterns. The new formulas will be a new member of mathematical spiral equation family and have potential applications in architectures, industrious designs, artwork creations and descriptions of natural objects.
\end{abstract}

Keywords: Spiral Galaxies, Spiral Simulation, Galaxy Evolution, Black Holes, Hydrogen Production, X-matter Conversion

\section{Introduction}

If at first the idea is not absurd, then there is no hope for it - Albert Einstein. The mechanism of forming spiral arms of galaxies has been extensively studied through the history of astronomy since the discovery of the most fascinating objects in the sky. Our universe is full of different types of galaxies classified with Hubble sequence based on the morphology of the galaxies $[1,2]$. So far there is no satisfactory model developed yet and the mechanism of the formation of the spiral arm is still unclear. Mcvitte and Payne-Gaoschkin proposed a model based on the Newtonian gravitation laws
[3]. The density wave theory (DWT) is the current leading theory in explaining the formation of the spiral pattern proposed by Lin and Shu in 1964 [4, 5], it describes the spiral arm as a quasi-stationary density wave that rotates around the galactic center with a constant pattern speed, it is limited only to the spiral galaxies which have well defined architectural spiral arm patterns such as grand design galaxies, which are only a small portion of all types of galaxies, the DWT cannot explain the formation of spiral galaxies with spurs and branches. The swing amplification model describes the spiral arms as a superposition of many unstable waves, the spirals generated by the swing 
amplification are transient and recurrent [6]. Several other alternatives or theories from DWT such as Manifold theory, Magnetohydrogendynamics theory and N-body simulations were proposed to explain the formation of the spiral arms [7-10], those models can only be used for few subsets of the spiral galaxies, not for broad application. The universe evolves in all time scale, galaxies as one of the major components of the universe evolve in all time scale as well; the morphology of the spiral patterns of the galaxies changes in all time scale also. A universal model is strongly desirable to reasonably describe most (if not all) spiral galaxies, a completely new approach has to be used. In previous paper, the author proposed a new hypothesis with derived formulas to explain the formation of the spiral arms of the galaxies [11], 9 different theoretic spiral patterns were produced with those formulas and 8 real spiral arm pattern images of galaxies with different categories were precisely simulated; and Hoag's object was naturally explained by this model. In this paper, the application of the proposed new model will be extended to study selected 7 galaxies with very special spiral patterns, and the possible formation and evolution sequence of Hoag's object will be illustrated. Very interesting information is extracted from the spiral patterns of the galaxies.

\section{Brief Description of the New Model for the Spiral Arm Formation}

Please refer the reference paper for the detail of the proposed new model and derivation of the formulas for the spiral arm formation of the galaxies [11]. The new model is briefly outlined below:

1. The spiral galaxies can be treated as an ideal fluid system with flat differential rotation velocity beyond the bar rotation area.

2. A supermassive black hole is located at the center of the galaxy, which has extremely high mass density and gravitational force, it may absorb matter from surrounding by its strong gravitational force, X-ray is emitted when the matter is orbiting around the black hole in an accretion disk, at the same time, the matter inside of the supermassive black hole with its extremely unimaginable physical conditions is converted to new unknown matter with non-gravitational property or anti-gravitational property and the unknown matter is gradually accumulated, the internal pressure (quantum degeneracy pressure or other unknown pressure?) is also built up. When such pressure reaches a critical level, the supermassive black hole cannot hold it, the unknown matter is bursted/erupted, the unknown matter is emitted by the supermassive black hole through its two opposite directions in the galactic rotation plane similar to the light beams emitted by a lighthouse shown in Figure 1. The supermassive black hole becomes an emission-active black hole (analogy to active volcanos), the unknown matter with non-gravitational property or anti-gravitational property is gradually converted to hydrogens on its moving path.

3. The unknown matter moves in a straightly confined route for certain distance after leaving the central emission-active black hole, then, moves freely, the length of the confined route is the observed half length of galactic bar. The galactic rotation fluid will drag the unknown matter with it, therefore, the final motion of the unknown matter is the combination of its emission velocity and the galactic rotation velocity.

4. The hydrogens converted from the unknown matter temporarily increase the local hydrogen density, refuel local existing stars and promote new star formation. The local luminosity is temporarily enhanced which is the spiral arms and galactic bar observed.

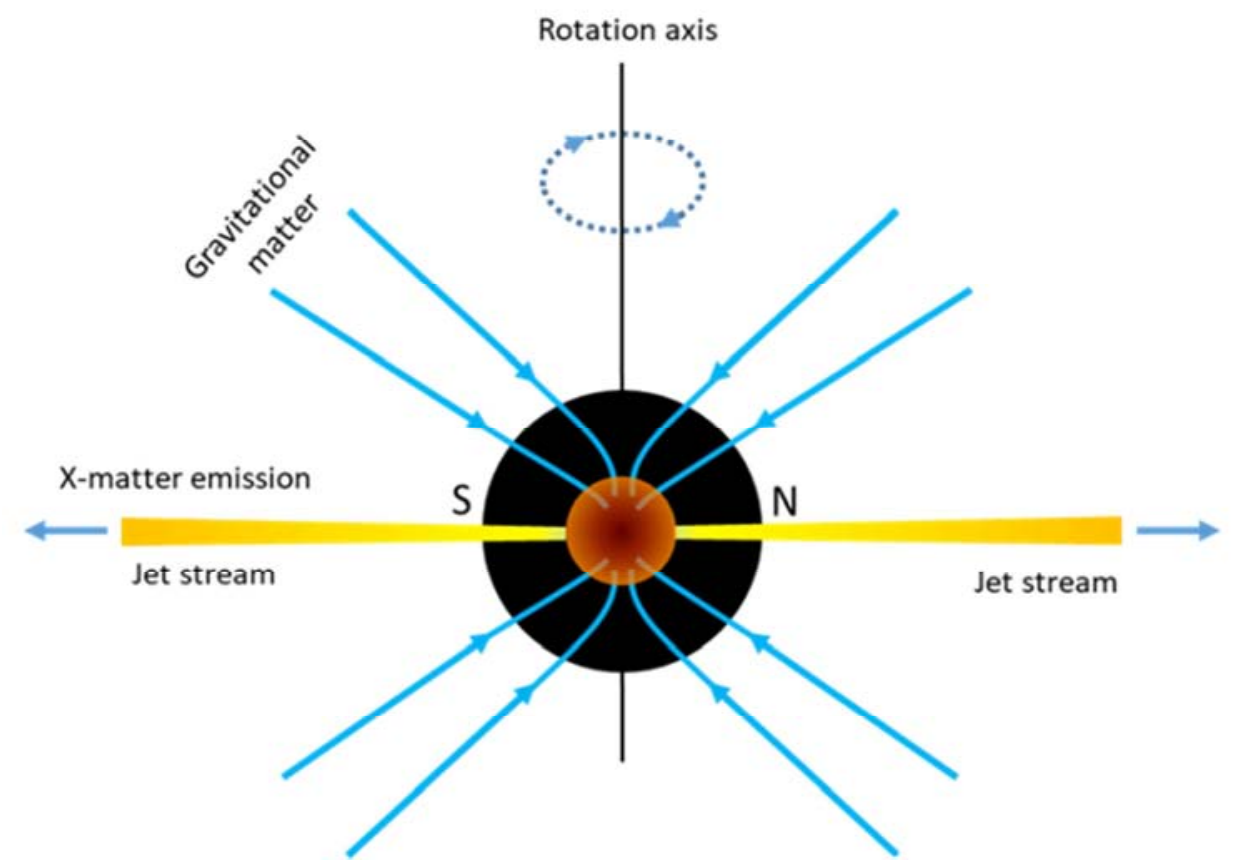

Figure 1. Illustration of unknown matter (X-matter) conversion and emission by the supermassive black hole located at the center of the galaxy. 
Treating the spiral galaxies as ideal fluid systems can be justified by morphology comparison of the spiral galaxies with hurricanes. In American Physical Society's 2004 Meeting of the Division of Particles and Fields held at the University of California, Riverside, the author proposed in oral presentation slide show that the fluid mechanics should be involved in the formation of galaxies based on the comparison of the morphology of galaxies with the hurricanes shown in Figure 2, the title of the presentation is "Application of fluid mechanics to dark matter" [12]. The Figure 2 left is the image of the hurricane Luis of 1995 and the right is the image of the galaxy M77.
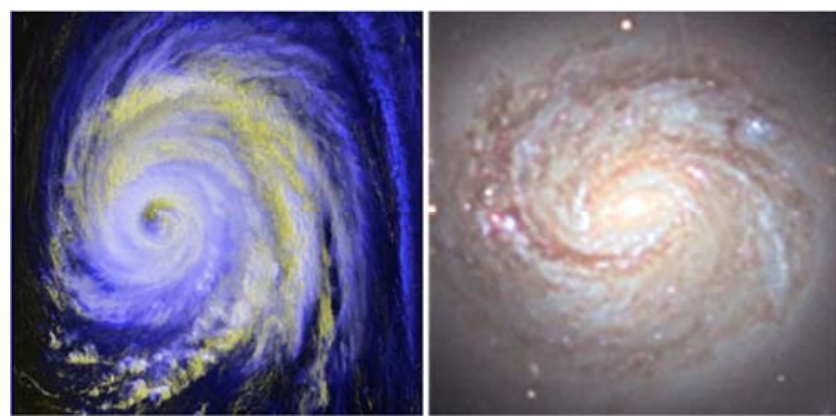

Figure 2. Left: Hurricane Luis, 1995; right: Galaxy M77.

The two complete different subjects have following common features:

1. They both have a flat disc-like shape. From the cosmic scale view (from the Earth), the morphology of the M77 galaxy is a small piece of rotating "cloud", the stars or star clusters and interstellar dusts act like water molecules or water drops and gases. The gravity may serve as the binding force and viscosity of the fluid.

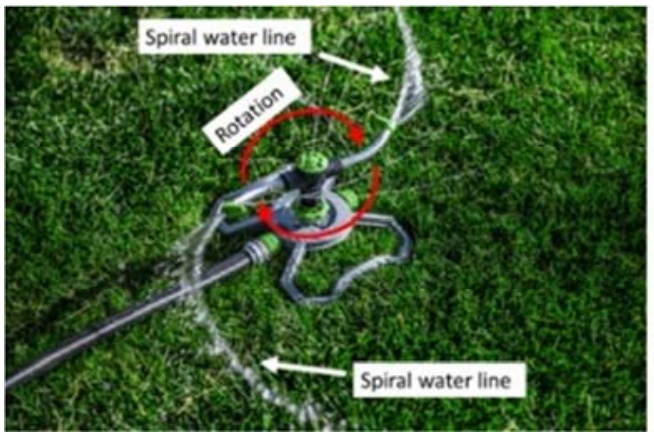

Figure 3. Left: 2-arm garden sprinkler watering the lawn with spiral water lines; right: Spiral galaxy NGC 4731.

The mechanism of the spiral arm formation outlined above is very similar to the mechanism of a 2-arm garden sprinkler watering lawn shown in Figure 3 left. The two spiral waterlines are symmetrical with respect to the center and are generated during the rotation of the sprinkler. Actually, the morphology and behavior of the spiral galaxy (spiral arms plus the galactic bar) is very similar to the waterlines of the 2-arm sprinkler, the morphology of the spiral galaxy NGC 4731 is "exact" as the 2-arm sprinkler shown in Figure 3 right, such similarity is the part of the inspiration for this model. The two completely different systems have the following common

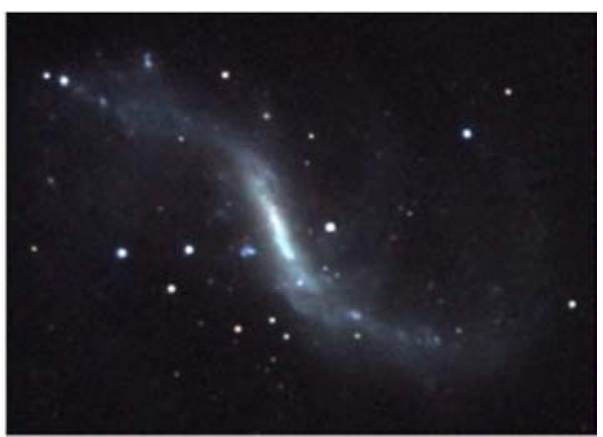

2. They both rotate around their centers.

3. They both have differential rotation velocity.

Therefore, the author believes that if two subjects look similar and act similarly, most likely, they shall share a same mechanism in great extent.

People commonly believe that all spiral galaxies have a supermassive black hole with millions of Sun's masses in the center of the galaxies. The current knowledge about the black holes is based on Einstein's general relativity. A black hole is a very small and perfect spherical object with extremely high matter density and gravitational force, it has only three externally observable classical parameters (no-hair theorem): mass, electric charge, and angular momentum, nothing within its horizon can escape from the black hole. The mass of the black holes varies dramatically, the mass of a black hole increases with surrounding matter accreting into it by its super powerful gravitational force; large black holes can be formed by merging also. Black holes in Star-Black hole binary systems have mass less than 30 times of Sun [13, 14]. Recently a new black hole was discovered in LB-1 of our Milky Way with estimated mass about 68 times of Sun [15]. However, the black holes should not be the end of the story although there are no theories or models to describe about the evolution of the black holes so far, the black holes as one type of cosmic objects should have their own evolution path after birth. In the proposed new model outlined above, the black hole located at the galactic center should be in a particular state of the black hole evolution sequence, when the black hole evolves to a critical stage, it starts to emit the unknown matter; some black holes may have different evolution routes and may not emit the unknown matter.

features:

1. For galaxies, spiral arms are always attached to the galactic bars (with exceptions which will be addressed later); The spiral waterlines are always attached to the ends of sprinkler arms (note this "arm" is the short tubing through which the water passes from the center to the nozzle, equivalent to the galactic bar).

2. Extensive research found that the spiral arms and galactic bars rotate together as a "rigid" body [16]. For the 2-arm sprinkler, the spiral waterlines and the 2-arms rotate together as a "rigid" body. 
3. The quality of the spiral arms of the galaxies decreases with length of the arms from the ends of the galactic bars. The quality of the spiral waterlines decreases with the length from the nozzles (gradually broken into pieces), such length is the age of the spiral waterlines after they are generated at the nozzles. For the spiral galaxies, the length of the spiral arms from the ends of the galactic bars should be the age of the arms also, this is a very important indicator and will be seen later when analyzing the real spiral arms of the galaxies.

Again, the author believes that if two subjects look similar and act similarly, most likely, they shall share a same mechanism in great extent.

In the previous paper [11], the model was named as Rotating Double-Side Sprinkle Emission model, for short, RDSSE model. However, this name may cause some confusion based on the readers' comments and not accurately reflects the nature of the model. In this paper, the proposed model will be named as ROtating Two Arm Sprinkler Emission model, for short, ROTASE model, and will be used through the paper. This name is easy in reading, accurate and no confusion. The unknown matter in the model outlined above is not the dark matter and dark energy, its property is totally unknown, it was temporarily named as "imaginary matter" in the previous paper due to lack of proper name at that time and may cause confusion also. Now it can be named as $\mathrm{X}$-matter, the " $\mathrm{X}$ " means unknown, which is similar to "X-ray" which was given for "unknown" when it was discovered by Wilhelm Röntgen in 1895 . The X-matter will be used through the paper.

\section{The Formulas to Calculate the Spiral Arm of the Galaxies}

Four mathematical formulas have been derived based the proposed model in the previous paper and are listed below, and the different spiral arm patterns of the galaxies can be calculated by those formulas and the real images of the spiral galaxies can be precisely simulated. Again, please refer the reference paper for the detail of the model and formulas derivation [11].

$$
r=\frac{R_{b}}{1-\rho * \sin (\alpha)}
$$

For $\rho>1$ :

$$
\begin{gathered}
\left(\frac{1}{\rho^{2}-1}\right)\left\{\sqrt{\left(\rho^{2}-1\right) r^{2}+2 r R_{b}-\left(R_{b}\right)^{2}}-\left(\frac{R_{b}}{\sqrt{\rho^{2}-1}}\right) \ln \left|r \sqrt{\rho^{2}-1}+\frac{R_{b}}{\sqrt{\rho^{2}-1}}+\sqrt{\left(\rho^{2}-1\right) r^{2}+2 r R_{b}-\left(R_{b}\right)^{2}}\right|\right\}-\left(\frac{R_{b}}{\rho^{2}-1}\right)(\rho- \\
\left.\frac{1}{\sqrt{\rho^{2}-1}} \ln \left|\frac{\rho^{2} R_{b}}{\sqrt{\rho^{2}-1}}+\rho R_{b}\right|\right)=R_{b} \theta
\end{gathered}
$$

For $\rho=1$ :

$$
\frac{\sqrt{2}}{3 \sqrt{R_{b}}}\left(r+R_{b}\right) \sqrt{r-\frac{R_{b}}{2}}-\frac{2}{3} R_{b}=R_{b} \theta
$$

For $\rho<1$

$$
\begin{array}{r}
\frac{R_{b}}{\left(1-\rho^{2}\right)^{\frac{3}{2}}} \arcsin \left(\frac{\left(1-\rho^{2}\right) r-R_{b}}{\rho R_{b}}\right)-\frac{1}{\left(1-\rho^{2}\right)} \sqrt{2 r R_{b}-\left(R_{b}\right)^{2}-\left(1-\rho^{2}\right) r^{2}} \\
-\frac{R_{b}}{\left(1-\rho^{2}\right)^{\frac{3}{2}}} \operatorname{acrsin}(-\rho)+\frac{\rho R_{b}}{1-\rho^{2}}=R_{b} \theta
\end{array}
$$

The value of arcsin is in unit of radian not in degree. $r$ is the distance of spiral arm in the calculation to the galactic center. $\alpha$ is the angle of $r$ with respect to the $\mathrm{X}$-matter emission axis. $R_{b}$ is the half-length of the galactic bar. The parameter $\rho$ is defined as the ratio of the emission velocity $V_{e}$ of the $\mathrm{X}$-matter out of the galactic bar over the flat rotation velocity $V_{r}$ of the galactic disc:

$$
\rho=\frac{V_{e}}{V_{r}}
$$

The original differential equations have to be solved with three different $\rho$ cases: $\rho>1, \rho=1$ and $\rho<1$, respectively to give equations (2), (3) and (4). The $\theta$ is the galactic bar rotation angle from a defined reference axis in which the X-matter is out of the end of galactic bar; $\theta$ is equivalent to the evolution time scale $t$ by the following equation with assumption that the galactic disc rotation is stable for the related time period:

$$
t=\frac{R_{b}}{V_{r}} \theta
$$

Therefore, the galactic bar rotation angle $\theta$ can be used as time counting, equations (2), (3) and (4) synchronize the $\mathrm{X}$-matter movement not only with time and but also with galactic bar position. The relationship between $r, \alpha$ and $\theta$ is clearly illustrated in Figure 4. The spiral arms are generated during the bar rotation because the X-matter is continuously emitted. One can see that the formulas have only one parameter $\rho$ and one variable $r$; the $\rho$ can change in any format, i.e., the X-matter emission velocity can change in any format, different $\rho$ will produce different spiral patterns. The steps to calculate the spiral arms are outlined in the reference paper [11]. 9 theoretic spiral patterns with different categories were produced by the formulas; 8 real images of galaxies with different categories were precisely simulated in the reference 
paper (UGC12158, NGC2273, NGC1398, NGC1300, M51, UGC6093, NGC4622 and NGC 4731) [11], and will not be duplicated here to respect the copy right.

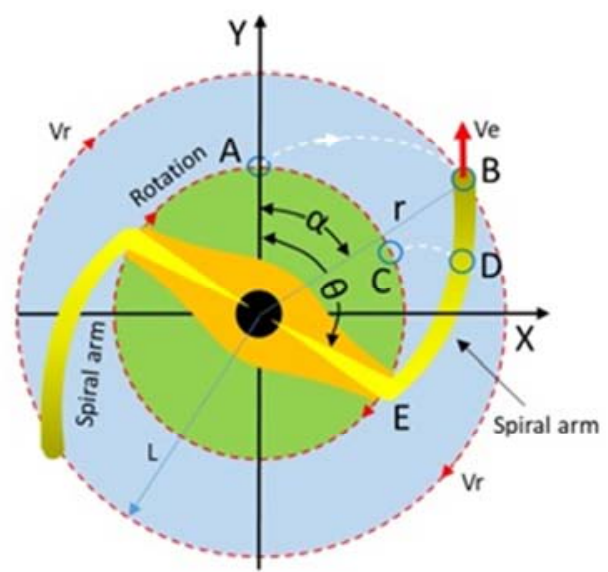

Figure 4. The relationship between $r, \alpha$ and $\theta$. Left: original position of the bar and X-matter is emitted at location $A$ with velocity $V_{e}$. Right: X-matter emitted at the A moves to location $B$ and still keeps it emission velocity $V_{e}$, and at the same time, the galactic bar rotates to location $E$.

\section{Luminosity and Quality of Spiral Arms Decrease with Time or the Length of the Arms}

In Figure 4, the green area is the bar rotation area which contains very dense old stars, the rotation velocity linearly increases with the radius; the light blue area is the flat rotation area in which the stars and interstellar dusts rotate around the galactic center with flat differential rotation velocity, and the star density decreases with radius. Figure 4 left is the original/start position of the bar, the X-matter exits the bar end vertically at location $A$ with velocity $V_{e}$, the emitted $X$-matter will move along the white dash line to location $\mathrm{B}$ with the combined motions of $\mathrm{V}_{\mathrm{e}}$ and flat rotation $\mathrm{V}_{\mathrm{r}}$ as shown in Figure 4 right.

During such motion, the X-matter is gradually converted to hydrogens. For simplicity, let us assume that the conversion rate follows the first order, i.e, the rate of X-matter converted to the hydrogens is proportional to the total amount of the remaining $\mathrm{X}$-matter:

$$
-\frac{d M(r, \alpha)}{d t}=K M(r, \alpha)
$$

Where, $\mathrm{M}(\mathrm{r}, \alpha)$ is the total remaining X-matter at location $(\mathrm{r}, \alpha), \mathrm{K}$ is the conversion rate constant. Then,

$$
M(r, \alpha)=M_{0} e^{-K t}
$$

$\mathrm{M}_{0}$ is the initial amount of the X-matter. The total amount of $\mathrm{X}$-matter decreases with time. The hydrogen production rate is proportional to the $\mathrm{X}$-matter conversion rate:

$$
\frac{d H(r, \alpha)}{d t}=k M(r, \alpha)=k M_{0} e^{-K t}
$$

Where $H(r, \alpha)$ is the amount of produced hydrogens at the location $(\mathrm{r}, \alpha), \mathrm{k}$ is the hydrogen production rate constant. The hydrogen production rate decreases with time. So, when the
X-matter travels to location $\mathrm{B}$, the extra hydrogen density produced by conversion of X-matter is less than hydrogens produced at location A along the motion line (white dash line), the stars near the motion line receive less and less extra hydrogens, so the enhanced luminosity decreases due to decrease of extra hydrogens along the motion line. The luminosity of the $\mathrm{X}$-matter traveled area will be back to normal after the X-matter passes through due to depletion of the extra hydrogens. The first order conversion assumption here is just for illustration purpose, the real case could be more complicate than the first order assumption. The X-matter is emitted continuously during the galactic bar rotation shown in Figure 4 right. When the bar rotates to location $\mathrm{E}$, the $\mathrm{X}$-matter emitted at location A already traveled to location $\mathrm{B}$, the $\mathrm{X}$-matter emitted at location $\mathrm{C}$ traveled to location $\mathrm{D}$, a line with enhanced luminosity is formed which is the observed spiral arm from B to D and E. However, the luminosity of the line decreases from $\mathrm{E}$ to $\mathrm{B}$, because the $\mathrm{X}$-mater at location $\mathrm{E}$ just exits the end of bar, the hydrogen production rate is higher than the location $\mathrm{B}$ according to equation (9), in other word, the arm at location B is older than arm at location E. The length of arm at location B to the location $\mathrm{E}$ also represents the age of the arm. Apparently, arm at B location is older than arm at D location, so the quality of the arm decreases with the length of the arm. The star density decreases with radius, so, the star density at location $\mathrm{A}$ and $\mathrm{C}$ and $\mathrm{E}$ is higher than location $\mathrm{B}$, this makes the luminosity of the arm at B decreases even further. The quality of the arm even decreases further by fluid diffusion, spreading and local inhomogeneity of the fluid system such as vortex, nonuniform mass density and velocity, so the spiral arm will fade away when the spiral arm is long enough, this is why only less 2 spiral windings are commonly observed. It has to be clearly understood that the age of arm is not the age of the stars within the arm, the two ages are totally unrelated. The X-matter does not affect the galactic disc star distribution and rotation, this agrees with observation that the star density in the arm areas is the same as other areas with the same radius. After the X-matter is emitted by the central emission-active black hole, it moves in a confined route for certain distance, during such motion, at the 
same time it start to gradually convert to hydrogens, those extra hydrogens refuel the local high dense stars around the confined route area and promote new star formation, the luminosity is enhanced which is the observed bright galactic bar. Please understand that the star density in the bar area is similar to the star density in other area with the same radius, only the luminosity in the bar area is higher than other area.

\section{Application of the Model to Selected Galaxies with Very Special Spiral Patterns}

\subsection{MCG+00-04-051 with Arms Broken Connection with Galactic Bar Ends}

In this model, the spiral arms are observed due to the

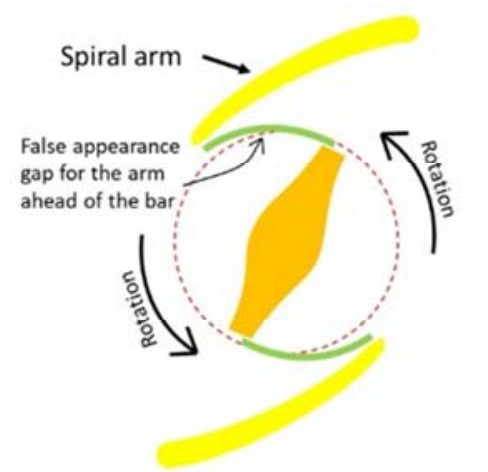

X-matter emitted from the central emission-active black hole, then converted to hydrogens which refuel the local stars during its motion and enhance their luminosity. This model predicts that when the X-matter emission stops, the arms will disconnect from the ends of the galactic bar, the bar still rotates and the arms still swirl outwardly, rotation gaps between the arms and the ends of the bar occur, such gaps increase with bar rotation. However, in previous study by Seigar and James [17], such gaps were believed due to the arms moving (rotating) ahead of the bar as illustrated in Figure 5 left (plotted in anticlockwise rotation). The new ROTASE model gives completely different explanation; the actual case may be that the arms are behind the galactic bar rotation as illustrated in Figure 5 right due to termination of X-matter emission.

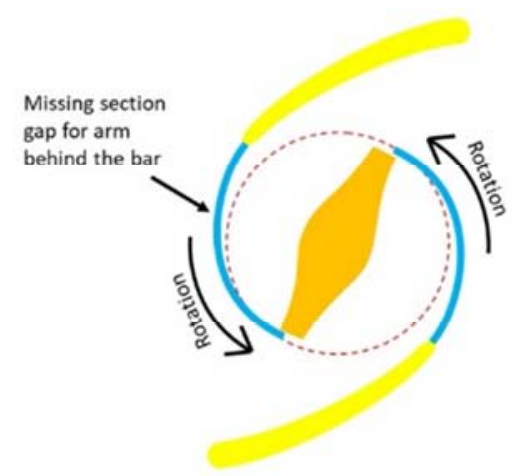

Figure 5. Left: false appearance of arms ahead of the bar; right: arms behind the bar.

The above prediction and Figure 5 were made in the first version of this model and published in October of 2019 where the figures were plotted with clockwise rotation. However, no clear image of the spiral galaxy with such scenarios could be found by the author at that time of publication to verify such prediction. Fortunately, the author recently found through internet by extensive searching that the spiral pattern of the galaxy MCG+00-04-051 perfectly matches the prediction of the ROTASE model, such perfect matching is the critical evidence as solid support for this model. It will be studied in detail here. Figure 6 left is the original image of the spiral galaxy MCG+00-04-051, it is also called SDSS J011430.80+001928.3.
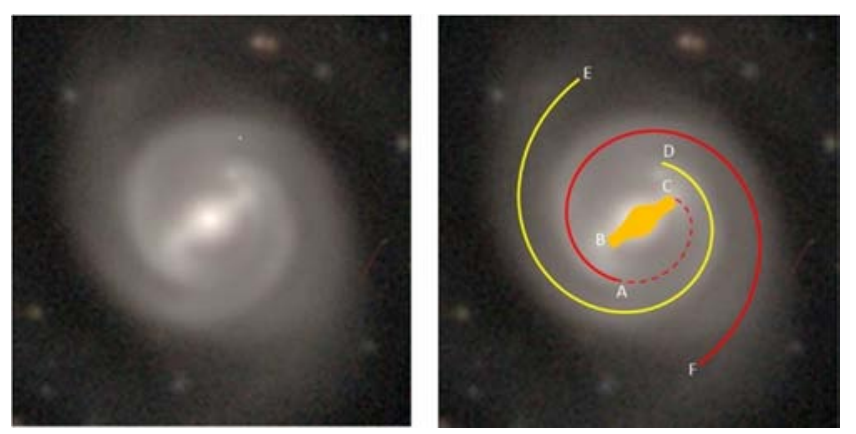

Figure 6. Left: the original image of galaxy $M C G+00-04-051$; right: Simulation by the ROTASE model Euler (-57, 0, 0).
This image is a face-on image with spiral arms having a perfect symmetry with respect to the galactic center. It clearly shows the disconnection of the arms from the ends of the galactic bar. The image is precisely simulated by the formulas (1) and (4) shown in Figure 6 right with the following parameter $\rho$ equation:

$$
\rho=0.08 *(1+0.015 * \theta)
$$

The galactic bar rotation angle $\theta$ in equation (10) represents the time defined by the equation (6), this is exact the same as a clock in which, the rotation angle of the needles is equivalent the time elapses, i.e., every 5 degrees of rotation of the long needle is the same as 5 minutes of time. The $\rho$ changes with time not the " $\theta$ ". The backward increase of the parameter $\rho$ with $\theta$ means that the $\rho$ was greater in the past, it continuously decreases from past to current direction. For convenience of the description, in Figure 6 right, A and D mark the inner ends of arms, which are symmetric with respect to the galactic center, $\mathrm{B}$ and $\mathrm{C}$ mark the ends of galactic bar, the $\mathrm{E}$ and $\mathrm{F}$ mark the ends of arm simulation, total rotation angle in the simulation is 550 degrees. In the ROTASE model, all arms are formed from emitted X-matter at the ends of bars, then, swirl outwardly by the combined motions of the velocity of the emitted X-matter and the flat rotation velocity of the galactic disc. Look at the red line arm of the Figure 6 right, the arm was formed from $\mathrm{F}$ to $\mathrm{A}$ in time sequence, at the time when $\mathrm{A}$ was 
formed at the end $\mathrm{C}$ of the bar, the $\mathrm{X}$-matter emission dramatically reduced, but the emission was not completely terminated, there was still very small amount of X-matter emitted from the end $\mathrm{C}$ of the bar, so the arm continued to be formed, which are evidenced with dramatically reduced but still visible spiral arm from $\mathrm{A}$ to $\mathrm{C}$ in the original image and as simulated by the thin red dash line, the red line from $\mathrm{F}$ to $\mathrm{C}$ is precisely calculated by equations. However, for the yellow line spiral arm, the arm was formed from $\mathrm{E}$ to $\mathrm{D}$, it was terminated at the $\mathrm{D}$ without continuing to the end $\mathrm{B}$ of the galactic bar, this indicates that the $\mathrm{X}$-matter emission at $\mathrm{D}$ was completely stopped or at least the arm in this period is so weak and not visible in this image. So the weak spiral arm section $\mathrm{AC}$ is the clear key support for the ROTASE model and proves that the arm end $\mathrm{D}$ is behind the bar end $\mathrm{B}$, not ahead of the bar end $C$, although, the distance from $D$ to $C$ is much smaller than the distance from $\mathrm{D}$ to $\mathrm{B}$, and gives a false impression that $\mathrm{D}$ is ahead of C. Broken connections at A and D happened at the same time and must be caused by internal activity, not by other celestial objects beyond the galaxy, because if it was caused by other objects, then, it would require two external objects to approach the galaxy in two opposite directions with the same intensity at the same time, such synchronized and collaborated impacts cannot happen in reality in such cosmic scale, or it can happen with zero possibility; furthermore, the external impacts should destroy the perfect spiral arms FA and ED. Therefore, the morphology of this galaxy can be reasonably explained only by the ROTASE model at this time, all other models cannot explain the morphology of this galaxy.

\subsection{NGC 4548 (M91) with Arms Broken Connection with Galactic Bar Ends}

The galaxy NGC 4548 is also called M91, classified as SBb (rs). This is a very interesting galaxy. Figure $7 \mathrm{a}$ is the original image with low quality.
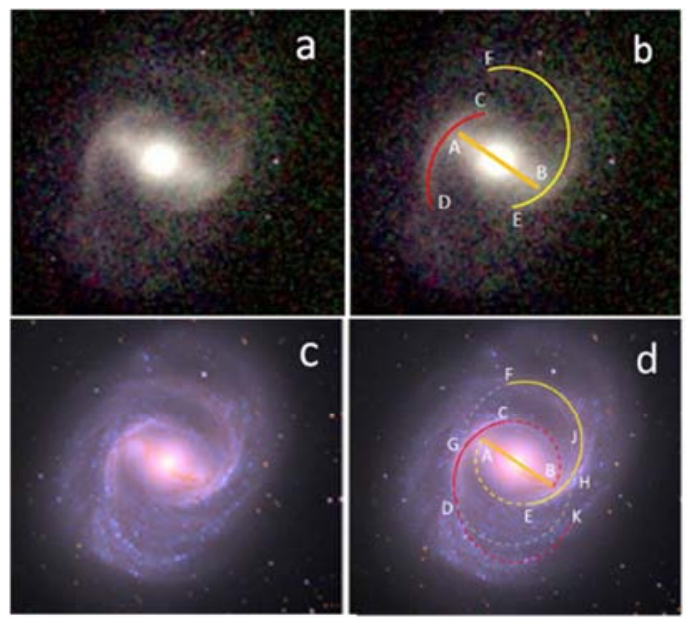

Figure 7. Images of Galaxy NGC 4548 and simulations.

a: the original image with low quality;

b: simulation by the ROTASE model, Euler $(20,15,20)$,

c: original image with high quality,

d: simulation by ROTASE model, Euler $(20,15,20)$.
In general, the spiral arm of galaxies intends to bind to the ends of galactic bars. Based on this low-quality image Figure $7 \mathrm{a}$, it is apparent that the spiral arms are not connected to the galactic bar ends, such pattern will give false impression that the arms rotate ahead of the bar rotation. The inner end points of the arms are labeled by letters $\mathrm{C}$ and $\mathrm{E}$ respectively in Figure $7 \mathrm{~b}$, points $\mathrm{C}$ and $\mathrm{E}$ are symmetric with respect to the galactic center which means that such disconnections happened at the same time by internal change not by outside impact from other celestial objects beyond the galaxy. The visible arms with well-defined profiles are simulated by red line CD and yellow line EF in Figure 7b with equation (1), (4) and the following parameter equation with Euler (20, 15, 20):

$$
\rho=0.52 * \exp \left(-0.0001 *(\theta-380)^{2}\right)
$$

Figure $7 \mathrm{c}$ is the original image of NGC 4548 with high quality. It clearly shows that the missing arm section $\mathrm{BC}$ in Figure $7 \mathrm{a}$ are visible in this image with dramatically reduced intensity, this reduced arm section $\mathrm{BC}$ as the red dash line in Figure $7 d$ clearly proves that the red line arm $C D$ was generated at the bar end $\mathrm{B}$, the $\mathrm{CD}$ arm section rotates behind the bar end B not ahead of bar end A. The main characteristics of Figure 7c is compatible with MCG+00-04-051.

Figure $7 \mathrm{~d}$ is the simulation. All important points are labeled by letters for description convenience. The red line BCGD and yellow line AEHJF are calculated by the equations (1, (4) and the parameter equation (11) with Euler $(20,15,20)$, and the red dash line DK is calculated by the following equation:

$$
\rho=0.6 * \exp \left(-0.00012 *(\theta-390)^{2}\right)
$$

The reduction of the arm $\mathrm{BC}$ is due to the reduction of $\mathrm{X}$-matter emission by the central emission-active black hole. The $\mathrm{BC}$ red dash line arm is calculated with 230 degrees of bar rotation time, the red line $\mathrm{CD}$ is calculated with 110 degrees of bar rotation time.

For the yellow line spiral arm, the invisible arm section AE in Figure $7 \mathrm{a}$ is barely seen in Figure $7 \mathrm{c}$ and shown by yellow dash line $\mathrm{AE}$ in Figure $7 \mathrm{~d}$, this means that the $\mathrm{X}$-matter emission in this period almost stops. The arm extends from $\mathrm{E}$ to $\mathrm{J}$, and then to $\mathrm{F}$, at the location $\mathrm{J}$, the arm seems slightly distorted. The JF section is calculated with 60 degrees of the galactic bar rotation time. The yellow arm beyond the point $\mathrm{F}$ is not well defined; the gray dash line FG is just the theoretical calculation of the extension of the yellow line by equation (11).

For the red line spiral arm, it starts at the bar end B, extends to $\mathrm{C}$ with clearly visible arm section $\mathrm{BC}$ with dramatically reduced luminosity due to the dramatically reduced $\mathrm{X}$-matter emission. The red CD section is the same as EJ of the yellow line arm; both are clearly visible in low quality image Figure 7a. The point $\mathrm{J}$ and point $\mathrm{D}$ are symmetric with respect to the galactic center. However, the red line arm section DK with 100 degree of galactic bar rotation time beyond the point D follows the equation (12), not the equation (11) which is the gray dash line $\mathrm{DH}$. This indicates that the $\mathrm{X}$-matter emission 
of the emission-active black hole in $\mathrm{DH}$ section is different from the yellow section JF, this means that inside of the emission-active black hole is highly active with non-equal emission at two opposite directions.

The X-matter emission at two directions by central emission-active black hole is not equal for most of time in this image; the emissions in both directions are (roughly) equal only in CD and EJ sections. The overall impression of the patterns of DB plus EF is the very similar to MCG+00-04-051 galaxy, and can only be explained by ROTASE model at this time.

Figure 8 shows three galaxies with arms not binding to the ends of galactic bar ends. It seems that such morphology is very common in the universe.
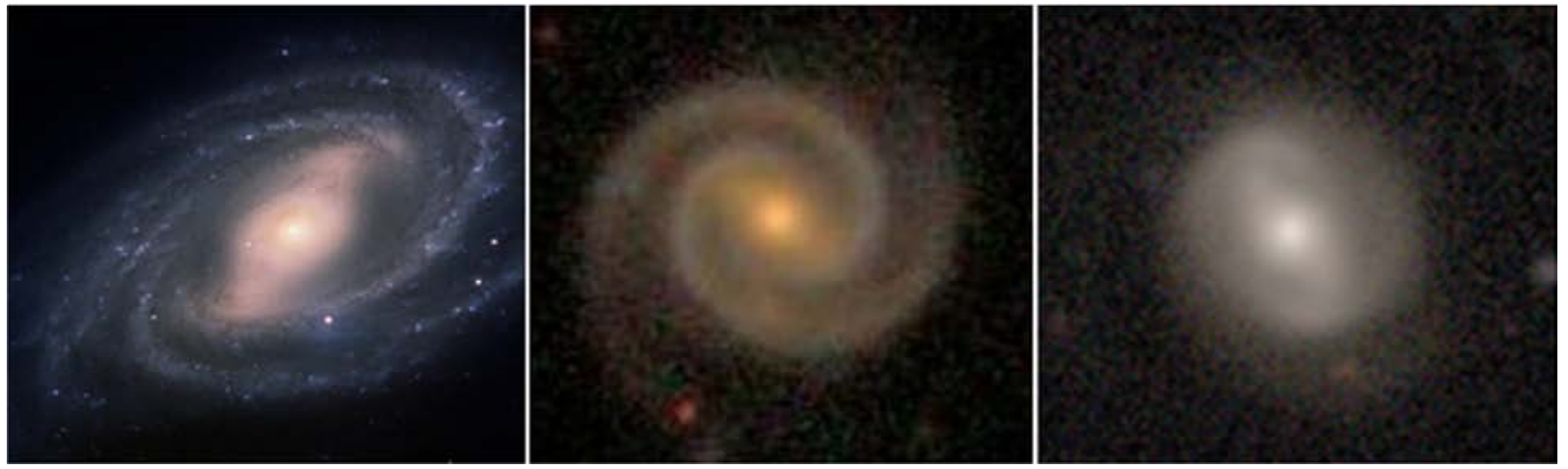

Figure 8. Three galaxies with apparent broken connection of arms with galactic bar ends Left: M109; middle and right: Unknown name galaxies.

\subsection{ESO325-28 with Perfect Spiral-Ring Pattern}

This galaxy shows a nice spiral-ring pattern, same as NGC 2273 [11], the original image is shown in Figure 9 left, which is face-on image. The profile of the spiral arm pattern was depicted by Buta shown in Figure 9 middle. This ring pattern was classified by Buta as R2' type where the arms wind $270^{\circ}$ with respect to the bar ends $[19,20]$. The simulation by formulas (1) and (4) with constant parameter $\rho=0.65$ is shown in Figure 9 right. The constant $\rho$ means that the central black
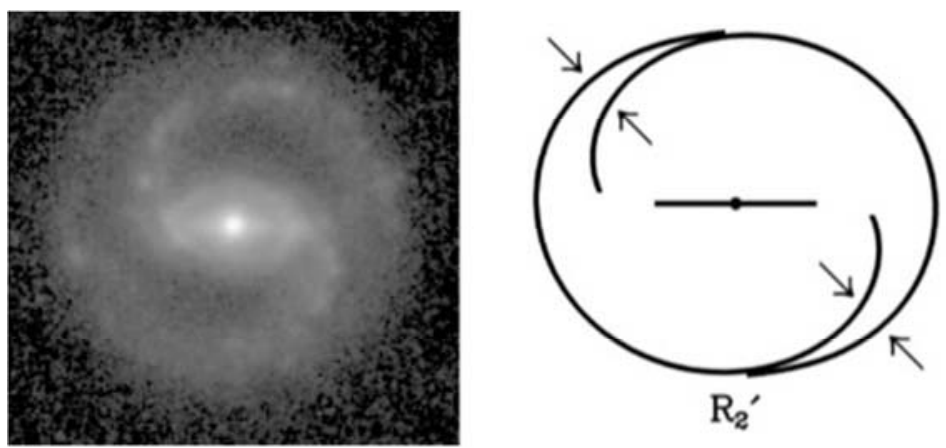

hole emits the X-matter at stable rate which does not change with time during the time period of the galaxy development shown in this image. Actually, this galaxy has a simple and perfect round spiral-ring pattern. The spiral-ring pattern has been addressed in detail in the reference [11]. For galaxies with $\rho<1$, the radius of the spiral-ring pattern is the maximum distance of spiral arms to the center of galaxies by the following equation:

$$
r(\text { radius of ring })=\frac{R_{b}}{1-\rho}
$$

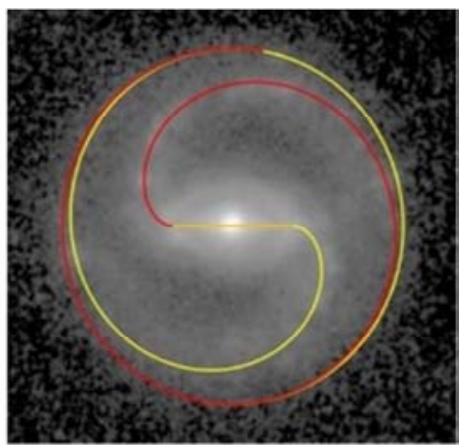

Figure 9. Left: original ESO325-28 image; middle: the depiction of the spiral arm profile by Buta; right: the simulation by the ROTASE model.

The number of spiral arm windings increases with $\rho$, but the gap between the spiral arms decreases from inner arms to outer arms as clearly shown in Figure 9 of the reference [11], i.e., the winding becomes tighter and tighter with increase of the spiral windings. In reality, only less than 2 windings are normally observed. For $\rho=1$, the ring size is infinitive, i.e., no ring pattern.

\subsection{NGC 7098 with Double-Rings with Chain-link Twice Arm Crossings}

Figure 10a is the original image of the NGC 7098 galaxy, classified as (R) SAB (rs) a. The morphology of this galaxy shows very beautiful double ring pattern, it is described in Wikipedia website as a smaller inner ring wrapped around the galactic bar and a big well-defined outer ring located outside of the inner ring. Such description is possibly the most accepted description in the astronomy community.

However, if we carefully inspect the image, we can find that the inner ring and outer ring are connected at the areas of galactic bar ends shown in Figure $10 \mathrm{~b}$ marked by red and yellow lines, such connections have very smooth spiral characteristics connecting the inner ring to the outer ring, and is highly symmetric with respect to the galactic center. Such special connection clearly reveals how the outer rings are developed. 

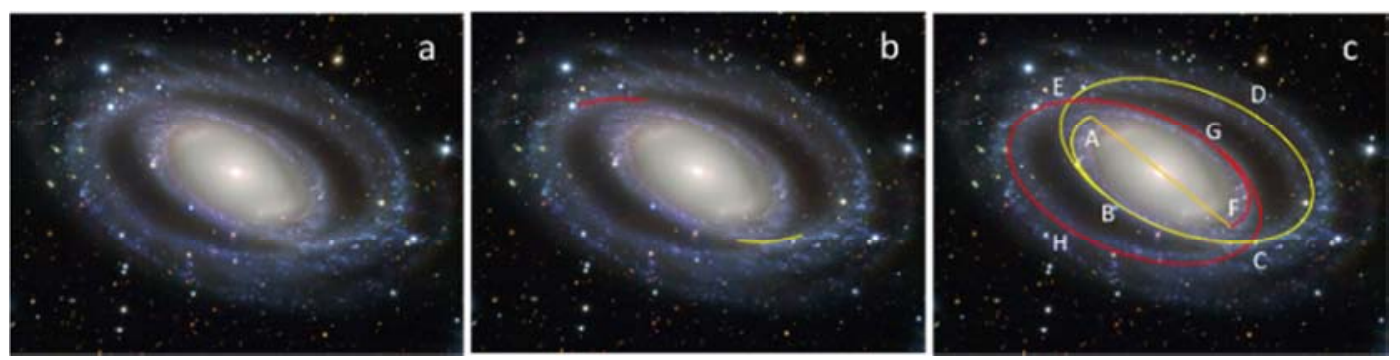

Figure 10. Image of NGC 7098 and simulation.

a: Original image of NGC 7098.

b: connection of inner ring to outer ring.

c: simulation by ROTASE model, Euler $(25,60,25)$.

Based on the ROTASE model, the description of the morphology of this galaxy will be completely different from the description in the Wikipedia website. The special double ring pattern can be precisely simulated by the equations (1) and (4) with parameter equation (14) shown in Figure 10c, the parameter $\rho$ changes with time which is equivalent to change with the rotation angle of galactic bar by the following Gaussian equation:

$$
\rho=0.55 * \exp \left(-0.0001 *(\theta-360)^{2}\right)
$$

Total rotation angle for the calculation is 540 degrees with Euler rotation $(25,65,25)$. The peak emission of the $\mathrm{X}$-matter was 360 degrees of bar rotation time in the past from the current time. The whole morphology of the NGC 7098 can be nicely described by the ROTASE model, all important points are labeled by letters in the Figure 10c for the convenience of description. Let's focus on the yellow line arm. The yellow line arm starts at the bar end $\mathrm{A}$, then, extends to $\mathrm{B}$ and reaches the point $\mathrm{C}$; at point $\mathrm{C}$, the yellow line arm crosses the red line arm. However, at the cross point $\mathrm{C}$, the yellow line arm is much younger than red line arm, therefore, the yellow line arm dominates (more visible) in this area. According to the ROTASE model, all spiral arms are generated at the ends of bars and move outwardly, the quality and luminosity of the spiral arms decrease with the age of the arm. The yellow line arm at point $\mathrm{C}$ was generated at 270 degrees of the bar rotation time in the past from current time. The red line arm at point $\mathrm{C}$ location was generated at other end $\mathrm{B}$ of the bar at 450 degrees of bar rotation time in the past from current time, so the red line arm at point $\mathrm{C}$ is 180 degrees of bar rotation time older than the yellow line arm at the same point, therefore, the yellow line arm has better quality and stronger luminosity at point $\mathrm{C}$. The yellow line arm continues to the point $\mathrm{D}$, then reaches the point $\mathrm{E}$, at which it crosses the red line arm again, but, at this cross point $\mathrm{E}$, the yellow line arm is 180 degrees of bar rotation time older than the red line arm, so the red line arm dominates at the cross point $\mathrm{E}$. The red line arm starts at bar end $\mathrm{F}$, and extends to GEHC points and has exact the same characteristics as the yellow line arm.

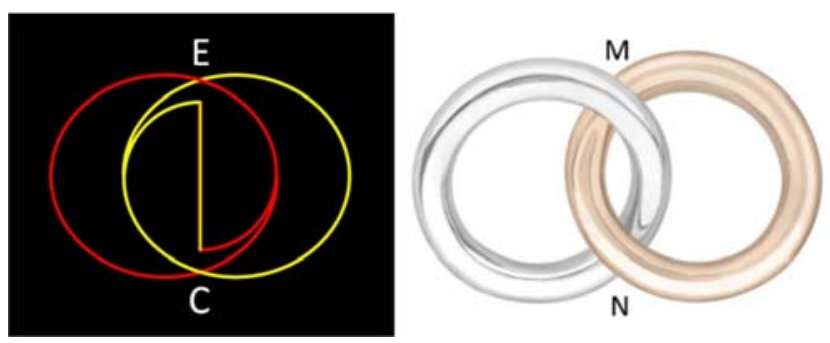

Figure 11. Left: the calculated face-on pattern of NGC 7098; right: Chain-link two rings.

Figure 11 left shows the calculated face-on spiral pattern of NGC 7098 with its galactic bar in vertical position. The yellow line arm at the cross point $\mathrm{C}$ looks like on the "top" of red line arm, because the yellow line arm is much younger than the red line arm at this point. At the cross point E, the red line arm looks like on the "top" of the yellow line arm. Such unique crossing style is very important for understanding how the outer rings are generated. Such ring crossing morphology is very similar to the pattern of the chain-link two rings shown in Figure 11 right, in which the gold ring is on the top of the silver ring at the cross point $\mathrm{N}$, and the silver ring is on the top of the gold ring at the cross point $\mathrm{M}$. Therefore, the special double spiral-ring pattern of NGC 7098 can be named as a chain-link double ring pattern. So, the special double ring pattern of this galaxy is actually made of two identical rings, each ring is made of a half inner ring and a half outer ring. Therefore, the description by ROTASE model is completely different from current commonly accepted description, it is more accurate and quantitative. Such double arm crossing is predicted in the previous paper that if the X-matter emission decreases with backward time, it will cause arm crossing, the spiral patterns will be more complicated if the X-matter emission in both increase and decrease direction in the history [11]. The double-crossing pattern of this galaxy completely matches such prediction. Other currently available models may not be able to explain this interesting unusual ring pattern.

The chain-link double ring galaxies are very common in the universe. The general parameter equation for this type of galaxies can be written as 


$$
\rho=\rho_{0} * \exp \left(-k *\left(\theta-\theta_{p}\right)^{2}\right)
$$

The $\rho_{0}$ is the maximum value of $\rho ; k$ is a constant controlling the width of the Gaussian profile; $\theta_{\mathrm{p}}$ is the angle (time) in the past from now, at $\theta_{\mathrm{p}}$ the X-matter has the peak emission. The parameter $\mathrm{k}$ in the equation (15) is very important, because it will significantly impact the morphology of the chain-link galaxies. Figure 12 illustrates how the value of $\mathrm{k}$ changes the morphology of the chain-link double ring galaxies.

Figure $12 \mathrm{a}$ is the current chain-link double ring pattern of
NGC 7098 with $k=0.0001$. Figure $12 b$ is the chain-link double ring pattern with the same equation with $k=0.00001$. One can see that the inner half rings $B$ and $G$ expand outwardly towards outer half rings $\mathrm{H}$ and $\mathrm{D}$, and the cross points $\mathrm{C}$ and $\mathrm{E}$ move outwardly, i.e, the two rings gradually merge. When $\mathrm{k}$ reduces to zero, the two rings are completely merged and become a perfect spiral-ring pattern shown in Figure 12c, actually, it is the two rings perfectly overlap together. So, the spiral-ring pattern is a special case of the chain-link double ring pattern and the radius of the ring is decided by the equation (13).
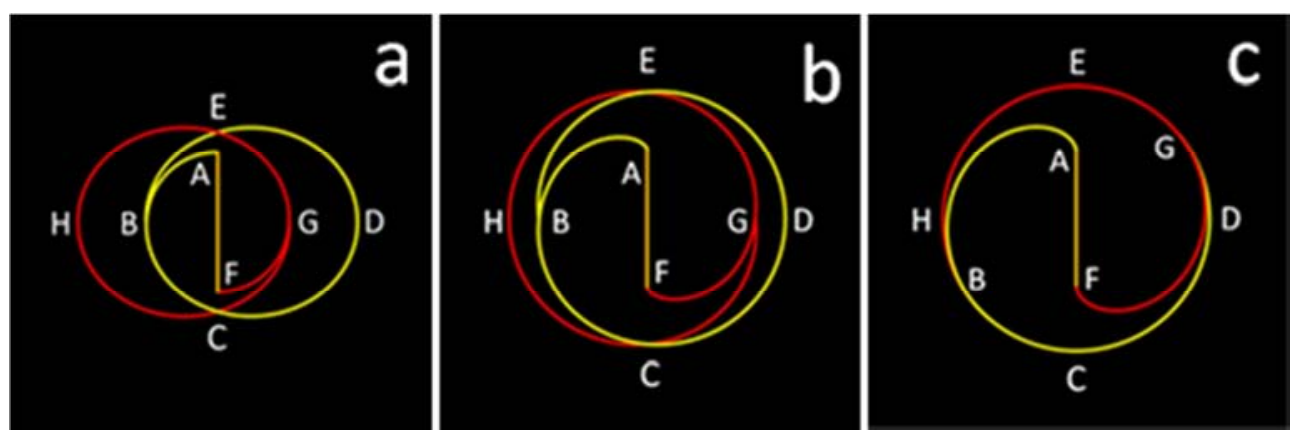

Figure 12. The chain-link double ring pattern changes with the Gaussian parameter $k$ in equation (15), $\rho_{0}=0.55, \theta_{p}=360 . a: k=0.0001 ; b: k=0.00001 ; c: k=0$.

\subsection{UGC 12646 - Double Rings with Chain-Link Twice Arm Crossings}

Figure 13 left is the original image of UGC 12646, the morphology of its unusual spiral-ring pattern was studied in detail by Buta, the profile of the pattern was depicted by Buta shown in Figure 13 middle. This galaxy is classified as type 1 OLR outer pseudoring R1' subclass by Buta where the outer arms wind $180^{\circ}$ with respect to the bar ends (a pseudoring from end to end) $[19,20]$. However, by careful examination, it is found that the arm does not wind from one bar end to another end. The unusual ring pattern can be precisely simulated by the formulas (1) and (4) with following parameter equation:

$$
\rho=0.655 * \exp \left(-0.00013 *(\theta-360)^{2}\right)
$$

Figure 13 right shows the simulation, the total rotation angle for the simulation is $550^{\circ}$. This image is not a face-on image.
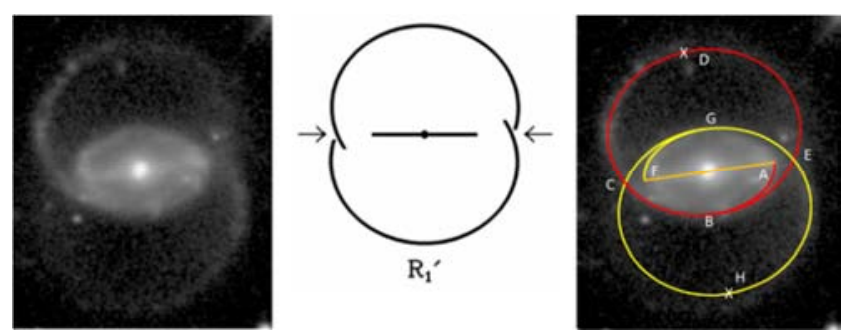

Figure 13. Left: original UGC12646 image; middle: the depiction of the spiral arm profile by Buta; right: simulation by ROTASE model, Euler (8, 50, $0)$.

For description convenience, all important points on the arms are labeled by letters. Look at the red line arm in Figure
13 right, it extends from bar end A to point B, C, D, E and back to $\mathrm{B}$. Based on the image, the distance of arm at point $\mathrm{C}$ to the bar end $F$ is the same as the distance of the arm at $E$ to the bar end $\mathrm{A}$, the DE section is much weak and is about to fade away. The quality of the red line arm at $\mathrm{C}$ is much better than red line arm at $\mathrm{E}$ which is almost faded away, such substantial difference in arm quality clearly indicates that the red line arm at point $\mathrm{E}$ is much older than red line arm at point $\mathrm{C}$. The quality of the red line arm gradually decreases from A to $\mathrm{E}$ along the arm line, such sequential quality decreasing in time matches the proposed model and is a good evidence to support this model, i.e., the quality of the arm decreases with its age. However, there is no correlation between the age of stars within the arms and the length of the arms. The age of stars in $\mathrm{C}$ area should be more or less the same as the stars in E area. The quality of the arm also depends on the quality of the disc such as the uniformity of the mass distribution and the flat differential rotation velocity of the disc. However, in this galaxy, the quality of the galactic disc is very good, the distance of the arms to the ends of bar may have no impact on the quality of the arms, otherwise, the arms would be deteriorated quickly by the severe inhomogeneity of the mass distribution and differential rotation velocity, no ring pattern would be observed like the galaxy NGC 1300 which only have a half ring spiral arm observed [11]. Such phenomenon is the result of the fluid dynamics, it is the same as the contrails made by jet airplanes or color smoke lines by airplanes during flying maneuvers, strong wind will destroy the patterns quickly. The same analysis holds for yellow line arm, the two ring arms cross each other twice at point $\mathrm{C}$ and $\mathrm{E}$, respectively. The age of the red line arm at point $C$ is much younger with better quality than the yellow line arm at the same point $\mathrm{C}$, so the red line arm dominates (more visible) in the $\mathrm{C}$ area of the 
image. At the cross point $\mathrm{E}$, the yellow line arm at $\mathrm{E}$ is much younger than red line arm, so the yellow arm dominates in point E area of the image. The pattern of UGC 12646 is the same as of NGC 7093.

Figure 14 shows the parameter $\rho$ changes with time (the rotation angle of galactic bar is equivalent to evolution time scale).

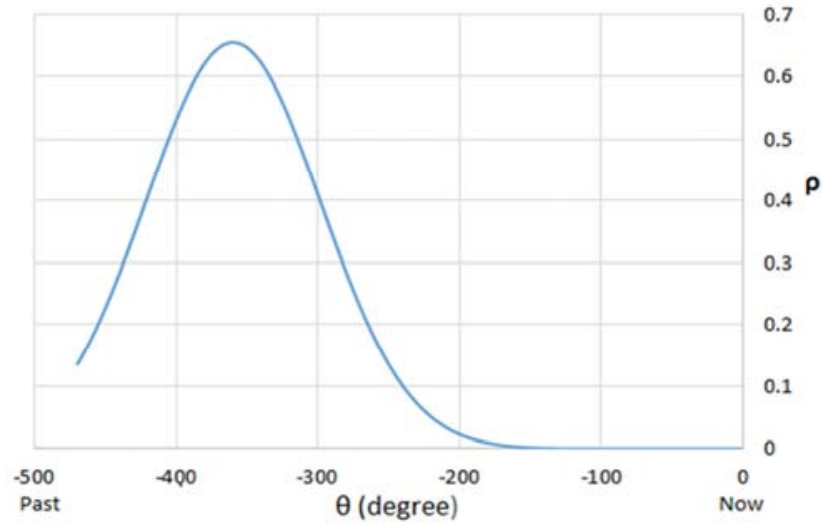

Figure 14. The parameter $\rho$ changes with time from past to current for the UGC 12646 (the bar rotation angle $\theta$ is equivalent to evolution time scale).

Remember, refer equation (5), the $\rho$ represents the X-matter emission velocity with assumption that the galactic disc rotation and the structure is stable during the related time period. Figure 14 may reveal a remote "tale" event in the past. Long before $550^{\circ}$ bar rotation angle time (possible several hundred million years ago), the matter accreted into the black hole located at the center of the galaxy, the X-matter was gradually accumulated inside the central black hole of the galaxy, the internal pressure was gradually built up also. When such pressure reached a critical level, the black hole could no longer hold the pressure, the X-matter erupted, the X-matter was emitted as shown in Figure 1, the black hole evolved to a new state and became an emission-active black hole. The $\mathrm{X}$-matter emission velocity gradually increased with time, it reached the peak velocity at $360^{\circ}$ galactic bar rotation time from now, marked by " $\mathrm{X}$ " at points $\mathrm{D}$ and $\mathrm{H}$ in Figure 13 right, then, the emission velocity has been gradually decreasing up to now, not suddenly change as galaxy MCG+00-04-051, each galaxy has its own specialty. It may stop emission at any moment in the future or continue such weak emission or increase again. Fortunately, the X-matter emission change with time follows the Gaussian style very well, gives such nice unusual double ring pattern with twice ring crossings, which can be nicely described by the proposed model. Figure 14 also indicates that the black hole is evolving in all time scale.

Back to the NGC 4548 galaxy, the parameter equation (11) is Gaussian function also, so if the X-matter emission of the central emission-active black hole followed the equation (11) well without reduction/stop and the galactic disc had good quality (good flat rotation velocity and mass distribution), the pattern of the galaxy NGC 4548 should be a chain-link double ring pattern shown in Figure $7 \mathrm{~d}$ with line AEJFG and BCDH like NGC 7098 and UGC 12646; the points G and H should be the arm cross points, the peak emission should be at 380 degrees of bar rotation time in the past from current time. However, such chain-link double ring pattern is not observed in this galaxy, the dash gray line arms DH and FG are not observed or at least, they do not have well defined (convinced) profiles; the dash gray line $\mathrm{AE}$ is not observed due to the termination of the X-matter emission. In summary, it is an incomplete or destroyed chain-link double ring pattern.

\subsection{NGC 1079 - Double Rings with Three Times Arm Crossing}

This galaxy shows the most amazing spiral pattern among the list of all galaxies studied in this paper and in previous paper [11]. Figure 15 left shows its original face-on image. The morphology of this galaxy was studied by Buta in detail [20], and the profile of the arm pattern of this galaxy is depicted by Buta as shown in Figure 15 middle.
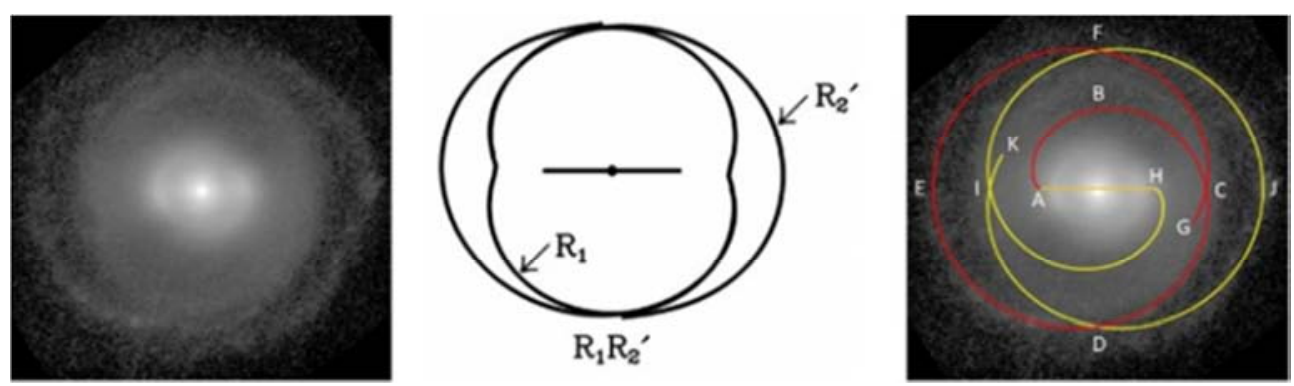

Figure 15. Left: NGC 1079, original image; middle: Spiral arm profile depicted by Buta; right: Simulation by ROTASE model.

This galaxy is classified by Buta as R1R2' subclass where the arms have mixed type 1 OLR outer ring R1 and type 2 OLR outer pseudoring R2' where the arms wind $270^{\circ}$ with respect to the bar ends. It shows multiple arm crossings. The first impression from the pattern profile is that multiple-section simulation has to be used to calculate the entire pattern, but with surprise, such complicated and beautiful pattern can be simply simulated by the formulas (1) and (4) with following parameter equation:

$$
\rho=0.655 * \exp \left(-0.00001 *(\theta-450)^{2}\right)
$$

The equation (17) is a Gaussian function. Figure 15 right shows the simulation, the total rotation angle of the galactic bar for the simulation is $650^{\circ}$. The increase and decrease of $\mathrm{X}$-matter emission is much slower than galaxy UGC12646, its peak emission velocity was at 450 -degree galactic bar rotation 
time from now. For description convenience, all important points on the arms are labeled by letters. Focus on the red line arm, it starts at galactic bar end A, extends to B, C, then crosses yellow line arm at point $\mathrm{D}$, extends to $\mathrm{E}$, then, crosses the yellow line arm at $\mathrm{F}$, then extends to $\mathrm{C}$ again, crosses itself at $\mathrm{C}$. The yellow line arm has the same characteristics. Each arm crosses other arm twice and crosses itself once. This is really a beautiful and unbelievable artwork by nature in such cosmic scale, and the perfect simulation with just one parameter equation is really a miracle by this model. Based this proposed model, the physical mechanism behind this artwork is quite simple, the X-matter emission just followed the right mathematical equation (17), and the image was captured luckily at the right time with the right angle.

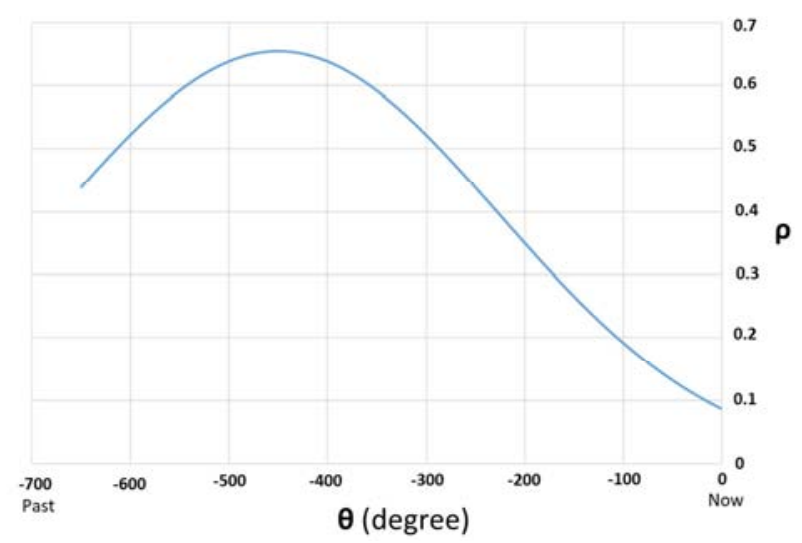

Figure 16. The parameter $\rho$ change with time from past to current for $N G C$ 1079 (the bar rotation angle $\theta$ is equivalent to evolution time scale).

Figure 16 is the parameter $\rho$ change with time from past to current. It is shown that the profile of the $\rho$ curve is much broader than the curve in Figure 14 of UGC12646. This galaxy is another good example to support the model. No other available models now and possibly in the future can make such precisely simulation.

Apparently, based on this proposed model, if the $\rho$ change is in "random" fashion, i.e., does not follow a well defined mathematical equation, the spiral pattern will be very complicated and possible featureless.
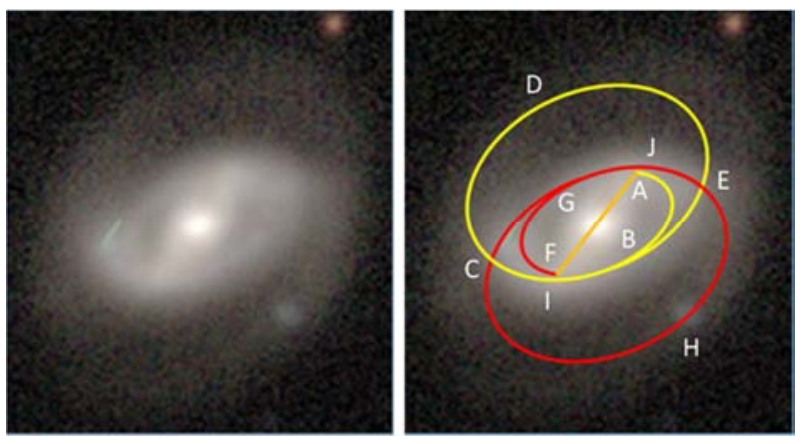

Figure 17. The original image of galaxy SDSS J015701.50-001644.4 (left). and simulation by ROTASE mode (right), Euler (-22, -55, 45).

\subsection{Galaxy SDSS J015701.50-001644.4}

The morphology of the galaxy SDSS J015701.50-001644.4 is very similar to galaxy UGC12646 but its bar rotated almost 90 degrees by a rough impression. Figure 17 left is its original image, this galaxy has several other names (2MASX J01570151-0016443, WISEA J015701.50-001644.4, etc.), its pattern can be precisely simulated by the ROTASE model shown in Figure 17 right by the following parameter $\rho$ equation:

$$
\rho=0.65 * \exp \left(-0.0001 *(\theta-410)^{2}\right)
$$

In this galaxy, the yellow line arm starts at galactic bar end $\mathrm{A}$, extends to $\mathrm{B}$ and $\mathrm{I}$, then crosses red arm at $\mathrm{C}$, then, extends to $\mathrm{D}$, then, crosses the red line arm again at $\mathrm{E}$, then extends to $\mathrm{B}$ to complete the ring, the entire yellow line arm contains a small half inner ring ABI and a large whole oval ring ICDEBI. The red line arm has exactly the same characteristics. Each oval ring crosses other ring twice with chain-link style. This is really a very impressive result by the ROTASE model. The peak emission of X-matter is at 410 degrees of galactic bar rotation time in the past from current time. The quality of the yellow line arm DE and the red line arm $\mathrm{HC}$ are poor, and almost fade away, this is because they are very old, such quality gradual decrease along the arm line from the ends of galactic bar fully agrees the ROTASE model.

If the galaxy SDSS J015701.50-001644.4 continues evolving in the same trend, the outer rings will fade away, only the inner ring will be visible, the pattern of the galaxy will be similar to IC 816.

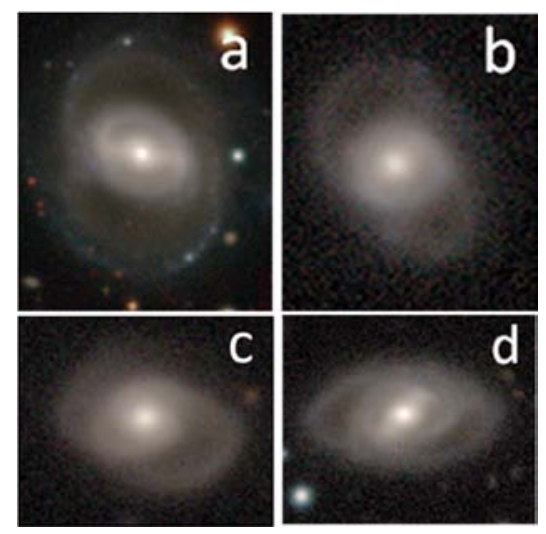

Figure 18. Four unknown-name galaxies with chain-link double ring morphology.

Figure 18 shows 4 unknown-name galaxies with chain-link double ring morphology. Such chain-link double ring galaxies are very common in the universe. Figure $18 \mathrm{a}$ is almost a copy of UGC12646. The Figure 18b shows special interesting pattern, the two outer half rings do not have equal size, one is much smaller than the other. This is due to unequal X-matter emission in the two directions by central emission-active black hole. Figure $18 \mathrm{c}$ looks similar to SDSS J105701.50-001644.4, but needs an image with better quality to verify this. Figure 18d looks a copy of NGC 7098.

\subsection{Possible Pattern Evolution of Hoag's Object}

Hoag's object is a very famous ring galaxy with a spherical bugle in the center without a bar structure as shown in Figure 
19c. Such interesting pattern was well explained by the ROTASE model [11].

Figure 19 illustrates the possible evolution sequence from Figure 19a to Figure $19 \mathrm{~b}$ to Figure $19 \mathrm{c}$ to Figure $19 \mathrm{~d}$ for the spiral-ring galaxy after the termination of X-matter emission by the central supermassive black hole.
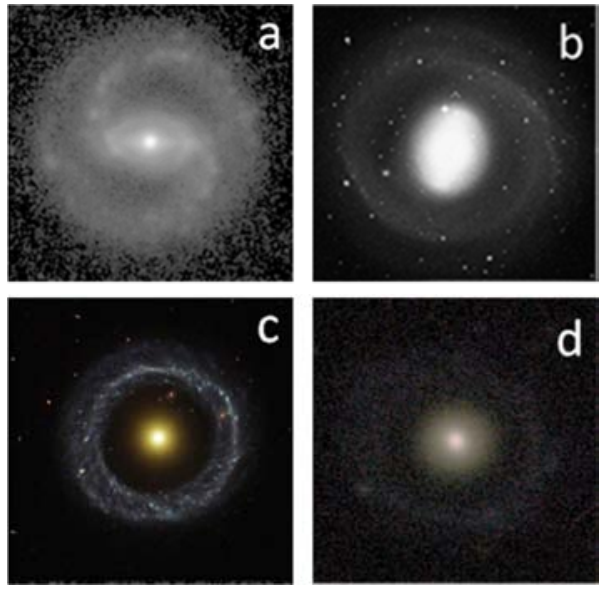

Figure 19. The possible evolution sequence of the galaxies with characteristics of Hoag's object after the termination of $X$-matter emission by the central supermassive black hole.

a: Spiral ring galaxy (ESO325-28) as original galaxy before the termination of X-matter emission. b: NGC 1291 as a possible example of the intermediate stage of the galaxy after the termination of X-matter emission, the galactic bar shrank with US football shape. c: Current Hoag's object

$\mathrm{d}$ : an unknown-name galaxy as a possible example of the evolution sequence with almost faded ring structure and dim central spherical bugle.

According to the ROTASE model, all spiral arms are formed by the stream of the X-matter emitted by central supermassive black hole. When the central black hole stops emission of the X-matter, it will cause broken connection of spiral arms with the ends of galactic bar, the spiral arms still swirl outwards and the galactic bar still rotates, the gaps between the arms and the galactic bar ends occur and gradually increase with time. This case has been studied in detail above in the galaxy MCG+00-04-051. The galactic bar will shrink due to depletion of the extra hydrogens converted by X-matter. If the original galaxy has a spiral ring structure like galaxy ESO325-28 shown in Figure 19a, when the central supermassive black hole stops $\mathrm{X}$-matter emission, the spiral arm portion continues to swirl outward and merge to the ring, the galactic bar will gradually shrink, the shape of the bar will become US football-like shape, the morphology of the galaxy at such intermediate stage will have a ring and a US football-like bar at the center, with gaps between galactic bar ends and the ring. The author found with extensive search through the internet that the galaxy NGC 1291 perfectly matches such intermediate morphology shown in the Figure 19b, it has a US football-like shrank galactic bar with big gaps from outer ring. If the galaxy evolves in the same trend, the galactic bar will continue to shrink and become a small spherical bugle in the center, which is the Hoag's object. If it continues such evolution, the central spherical bugle and the ring will get dimmer and dimmer, it will show the pattern like Figure 19d, the ring is barely seen; and will disappear in the future not long from now, the whole galaxy will be dark and invisible, but the galaxy is still there, only the luminosity dramatically decreases. Therefore, the universe should have a lot of dark/invisible galaxies wandering around and they will continue to evolve or collide, merge, and cluster with other galaxies.

\section{Pitch Angle of the Spiral Arms}

The pitch angle of spiral arms is one of the key important parameters in astronomy, it is a measure of the tightness of the spiral arms, i.e., how the arm is wound. The pitch angle $\phi$ is defined as the angle between the tangent line of the arm and the tangent line of the circle at the same point with center at the galactic center as illustrated in Figure 20.

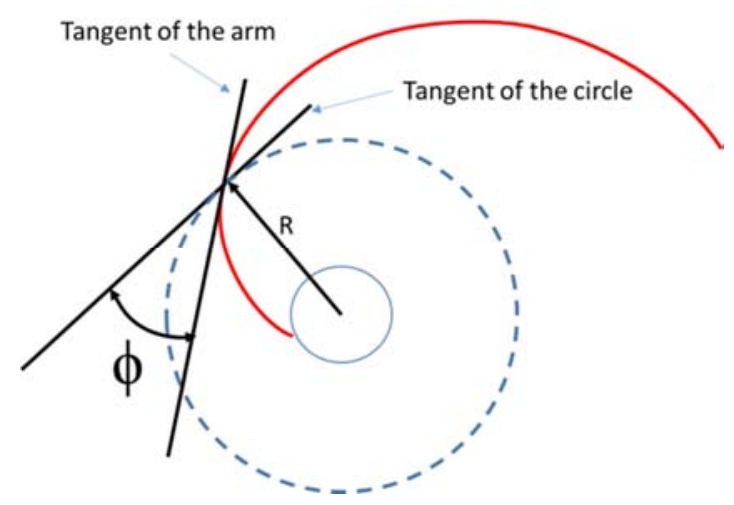

Figure 20. Pitch angle $\phi$ of a spiral arm.

To obtain the pitch angle, one has to use some mathematical formula to fit the arm pattern, the common formulas are logarithmic, Archimedean or hyperbolic spiral. However, those common formulas can only fit limited number of spiral galaxies. Most of the galaxies do not match those formulas.

The formulas from ROTASE model can simulate most of the spiral galaxies more accurately based on the results so far. Therefore, it will be much easier to obtain the pitch angle of the spiral galaxies by the formulas of the ROTASE model.

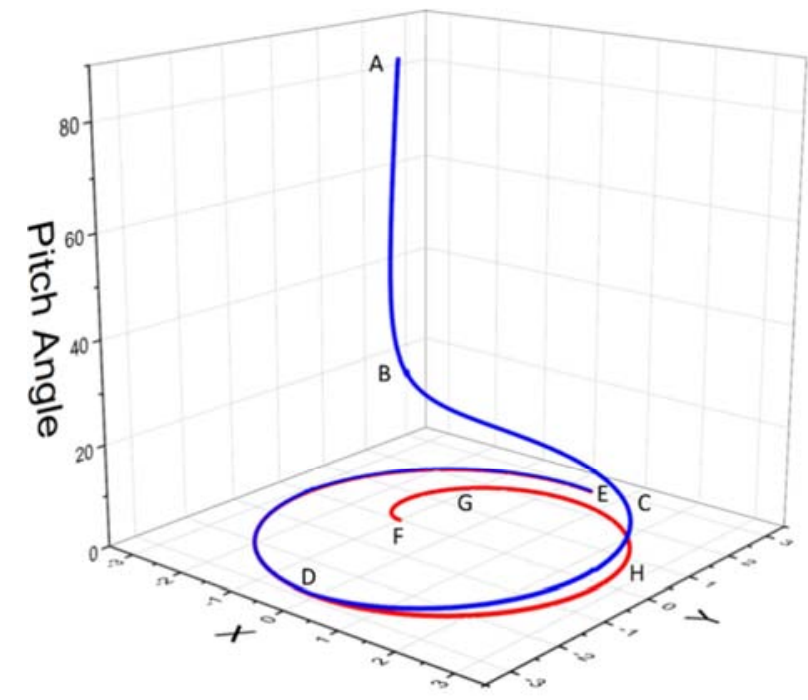

Figure 21. Pitch angle $\phi$ of one spiral arm of ESO325-28 galaxy. Blue line is the pitch angle; red line is one spiral arm of the galaxy. 
When the spiral pattern is nicely simulated by the formulas above, it will be very convenient to calculate the pitch angel of the spiral arms. Figure 21 shows the pitch angle of one spiral arm of ESO325-28 galaxy. The other arm has the same pitch angle due to perfect symmetry. Blue line is the pitch angle extending from A to BCDE; and the red line is the one arm starting at bar end $\mathrm{F}$ extending to GHDE. The pitch angle at bar end is almost 90 degree because the arm is almost perpendicular to the circle with radius of half length of the galactic bar. The pitch angle decreases quickly near the bar end range due to high curvature in the FG section of the arm. The pitch angle then gradually decreases to zero at point D which means that the spiral arm becomes a circle at point $D$ and after.

\section{Discussion}

One can see that the ROTASE model successfully and precisely simulated (together with previous paper) 15 spiral galaxies with substantially different types of morphology, such wonderful result proves that this model is a universal model and provides new formulas to simulate all (hope not exaggerated) types of spiral galaxies with a physical mechanism to explain how such patterns are formed. It also naturally explains some unusual galaxy patterns such as the Hoag's object, galaxies with broken connection between the arms and galactic bars because there is no good explanation for such patterns at current time by other models. Therefore, the ROTASE model is very promising, at least it shows a hopeful direction in this subject research. The simulation by ROTASE model is mathematically simple, the type of spiral patterns depends on only the behavior of the parameter $\rho$, i.e., how the X-matter is emitted by the emission-active black hole in the center of the galaxy. The Occam's razor should work here. The behavior of the $\rho$ may provide important information about evolution dynamics and history of the galaxies and the black holes at the centers of galaxies. Every black hole is different, therefore, each supermassive black hole at the center of a galaxy has its special X-matter emission behavior and make each spiral galaxy unique in the universe. The spiral arms and the galactic bars are the enhanced luminosity caused by temporary density increase of hydrogen gas produced from X-matter when the X-matter band passes the area in which existing local stars get refueled and new star formation is promoted with the extra hydrogens produced in those area, it is an optical effect; the galactic bars and the spiral arms together rotate as a whole rigid pattern. If the $\mathrm{X}$-matter emission changes with time, the spiral arm pattern will change with time. So far, all the extracted evidences from the morphology of the galaxies point to one thing: the spiral arms of the galaxy are generated at the ends of galactic bar through the mysterious matter (X-matter) emitted by the emission-active black hole located at the center of the galaxy.

The five galaxies (ESO325-28, NGC 7098, UGC12646, NGC1079 and SDSS J015701.50-001644.4) studied in this paper are a beautiful set of exquisitely crafted space artworks by the nature, each has its own unique beauty; and the
ROTASE model precisely duplicates those artworks after "peeking" at its construction blueprint. The success of the ROTASE model also demonstrates that the universe is operated by laws and mathematics is the execution tool of the laws; everything in the nature can be calculated by mathematics if we can successfully find the right formulas even the event looks very weird. A famous example in the history should be precise calculation of the "anomalous" precession of the perihelion of Mercury and the light bending in the gravitational field by Einstein's general relativity at 100 year ago and successful detection of gravitational wave few year ago; the most impressive observation is that a star circulates around supermassive black hole with the rosette-shaped orbit known as General Relativity Schwarzschild Precession [22].

Apparently, a logarithmic function cannot produce spiral-ring patterns; density wave theory or swing amplification model cannot describe such well defined multiple ring crossing patterns with sequential decrease of the quality of the spiral arms either. The dark matter and dark energy have been proposed for substantially long time, tremendous amount of resources and efforts have been put on searching such unknown matters, so far, no evidence was found for their existence, however, people still search for them. The proposed dark energy has anti-gravity repulsive force which causes the universe to expand at an accelerating rate. The proposed X-matter could be "similar" to the dark energy, because only matters with non-gravitational or anti-gravitational property can leave out of the supermassive black hole. There is no doubt that the new mathematical formulas derived from ROTASE model are correct, but the physical mechanism behind this model has to be tested. The proposed X-matter and central emission-active black hole seem quite absurd at the moment, can they be detected later or supported by other new theories in the future? One question has to be answered that: can right formulas be derived from a wrong or unreasonable physical mechanism? However, to author's limited knowledge, so far, no such successful example happened in the history of science, the ROTASE model could be the first one. The history of science did show that in many cases, as science and technology fast advance, today's non-sense could be tomorrow's common sense; the most famous example should be the Dirac's anti-particle hypothesis with which he was rewarded Nobel Price, no one believed him, people even laughed at him when he proposed his very weird hypothesis. The establishment of the quantum mechanics is another good example. Newtonian physics was a well-established theory at the end of 19th century, which could explain everything at that time, but the new experiment data indicated that the extremely small world must be "quantum" with event happening by probability, which was unacceptable by Newtonian physics, this caused the very famous debate between Bohr and Einstein. How can an electron pass two holes at the same time to interference itself? However, it is a magic, it is a fact, the God does play dice.

There are currently about 28 different mathematical spiral equations, the new formulas derived from ROTASE model 
will be significant in mathematics and will be a new member of the spiral equation family, which may have potential applications in architectures, industrious designs, artwork creations and descriptions of biological species and other natural objects with spiral patterns.

For almost all spiral galaxies, it is common observation that the spiral arms start from the ends of galactic bars with well-defined profiles and high quality, the quality of the spiral arms gradually decrease from the ends of galactic bars along the arm lines, the arms gradually spread and become looser and looser, then broken into pieces with more or less regular spaces between those pierces. A good example of such phenomenon is the galaxy NGC 1398. Such broken pieces with regular spaces were believed to be the direct observation of the Density Wave Theory [23]. However, it is just deterioration of the arms caused by fluid dynamics.

This model may not be perfect, may not explain the spiral arm formation of every spiral galaxy due to unexpected situations. However, it can be improved/modified in the future. There are currently no answers to the possible questions arisen from this model such as "how is the matter converted to $\mathrm{X}$-matter inside the emission-active black hole, then later is emitted and converted to the hydrogens?", "what is the confined route?", "does the X-matter have mass or it is massless?", etc. Extensive research in the future may provide answers, or may never have answers. Those questions are equivalent to questions "Why did the Big Bang happen?" and "How did the Big Bang happen?", there is no answer at this time. Currently, we just accept the "fact" that the Big Bang "did happen", because some of the observations match the possible consequences if the Big Bang "did happen". In Big Bang theory, the universe was created by a Big Bang at the singularity (we do not know where the singularity came from and if it was "real"), tremendous amount of matter was released, we do not know if such initial primary matter had mass or it was gravitational or non-gravitational or anti-gravitational. After some time later, such primary matter was converted to hydrogens, then, the stars, galaxies and the universe were formed. The conversion of X-matter to hydrogens could be a "mini version" of such process; this "mini version" does not mean that it is the same mechanism with smaller scale, because the physical conditions in the two scenarios are significantly different. One interesting question is that: was all primary matter from the Big Bang converted to Hydrogens and other materials shortly after the Big Bang? If it was, then, the current total amount of available hydrogens in the universe should be substantially less than the initial amount after 14 billion years consumption by formation of stars and burning, the available hydrogens will continuously decrease; and eventually, no new stars will be formed, the universe will be dimmer and dimmer, may contract to singularity again, start another universe cycle? However, based on the ROTASE model, the X-matter may drift away from galaxies and convert to hydrogens in the space, can be another source of hydrogens for star formations, or it may act like "dark energy"?

The formulas derived from this model have only one parameter and the one variable, therefore, they are the simplest formulas obtained in this subject for now and possible for the future, cannot be simpler (zero parameter or zero variable?). Such simplicity is fully consistent with Einstein's quotation "Everything should be made as simple as possible, but not simpler". The ROTASE model fully meets the two requirements for a good theory defined by Stephen Hawking [24]: A theory is a good theory if it satisfies two requirements. It must accurately describe a large class of observations on the basis of a model that contains only few arbitrary elements, and it must make definite predictions about the results of future observations. The success of the proposed model to describe the formation of the spiral arm of the galaxies is the result of the "bionics" borrowed from the hurricane and the 2-arm sprinkler, and bring up new questions: what is located at the center of the galaxy and what is really happening there? As Bertrand Russell said: The world is full of magic things patiently waiting our wits to grow sharper.

\section{Conclusion}

The result above is enough to justify the following conclusions:

1. The galactic disc is a rotating fluid system. The emission-active black hole in the center of the galaxy emits X-matter, the spiral arms come from X-matter. The $\mathrm{X}$-matter emission behavior of the emission-active black hole decides the spiral arm patterns. The black hole in the center of the galaxy evolves in all time scale; the information extracted by ROTASE model indicates that the property of the emission-active black hole is far more interesting and complicated than our current knowledge about the black hole.

2. The morphologies of the spiral galaxy MCG+00-04-051 and NGC 4548 perfectly match the ROTASE model. The weak but still visible arm sections provide critical evidence to support the model, the central emission-active black hole stopped X-matter emission, caused the arm disconnection from the ends of bar, such sudden change of X-matter emission reflects that the emission-active black hole was transferred to a different state, therefore, the behavior of the $\rho$ may be a good parameter to study the evolution of the central black hole. The relative positions of the inner arm ends with respective to positions of galactic bar ends will give a false impression that the spiral arm are ahead of bar rotation, however, in fact, the spiral arms are behind the bar rotation.

3. The ROTASE model reveals that the impression of double ring pattern of NGC 7098 with an inner ring and an outer ring is actually made of two identical rings and each ring is made of a half inner ring and a half outer ring, and each ring crosses other ring twice with chain-link style. The X-matter emission behavior by central black hole follows the Gaussian equation. The morphology of UGC 12646 has the same characteristics of NGC 7098. The quality of the arms decreases with age of the arm or 
the length of the arm from the galactic bar ends, which is the critical evidence to support the model. NGC 4548 shows incomplete/destroyed chain-link pattern due to very active central emission-active black hole.

4. The ROTASE model reveals that NGC 1079 has two identical rings, each ring has three times arm crossing: crosses other ring twice and crosses itself one once. This is the result of the X-matter emission following the Gaussian formula, which has a broader profile. The galaxy ESO325-28 is a perfect spiral-ring pattern. It is a special case of chain-link double ring pattern with perfect overlap of two rings.

5. The Galaxy SDSS J015701.50-001644.4 is made of two identical spiral structures, each structure is made of one big outer whole ring and a half small inner ring.

6. Hoag's object could be formed from a spiral-ring galaxy after termination of X-matter emission by central black hole and may become an invisible galaxy in the future.

7. The ROTASE model provides new mathematical formulas to simulate and analyze the spiral galaxies. The pitch angle of the spiral arms can be easily calculated after the successful simulation of the spiral patterns of the galaxies. The new formulas will be a new member of the mathematical spiral equation family, may have potential applications in architectures, industrious designs, artwork creations and descriptions of biological species and natural objects with spiral patterns.

\section{Acknowledgements}

This publication uses data generated via the Zooniverse.org platform, development of which is funded by generous support, including a Global Impact Award from Google, and by a grant from the Alfred P. Sloan Foundation. Specially thank to Ine Theunissen and Christine Macmillan for providing the information of the names of the Galaxy MCG+00-04-051 and Galaxy SDSS J015701.50-001644.4 through Galaxy Zoo Talk on Zooniverse.org.

\section{References}

[1] Hubble, E. P. (1927). The Classification of Spiral Nebulae. The Observatory. 50: 276.

[2] Hubble, E. P. (1936), Mrs. Hepsa Ely Silliman memorial lectures, 25. New Haven: Yale University Press. ISBN 780300025002 .

[3] McVitte, G., Payne-Gaoschkin, C., (1951). nA model of A spiral galaxy, MNRAS 111 (5), 506-522.

[4] Lin C., Shu F., (1964). On the spiral structure of disk galaxies. ApJ. 140, 646.

[5] Shu F., (2016). Six Decades of Spiral Density Wave Theory (2016)., Annu. Rev. Astron. Astrophys. 54, 667.
[6] Julian, W., A. Toomre, A., (1966). Non-axisymmetric responses of differentially rotating disks of stars, ApJ. 146, 810.

[7] Contopoulos, G., Grosbol, P., (1986). Stellar dynamics of spiral galaxies: nonlinear effects at the $1 / 4$ resonance, 1986, Astron. Astrophys. 155, 11-23.

[8] Contopoulos, G., Grosbol, P., (1988). Stellar dynamics of spiral galaxies: self-consistent models, Astron. Astrophys. 197, 83-89.

[9] Binney, J., Tremaine, S., 1987, Galactic Dynamics, (Princeton University Press, Princeton, NJ.

[10] Sellwood, J., Sparke, L., (1988). Pattern speeds in barred spiral galaxies, MNRAS. 231, 25-31.

[11] Pan, H., (2019). New Formulas and Mechanism for the Spiral Arm Formation of Galaxies, IJP, 7 (3), 73-85, DOI: 10.12691/ijp-7-3-2.

[12] Pan, H., (2005). Application of fluid mechanics to dark matter, Int. J. Mod. Phys. A., 20 (14), 3135-3137.

[13] Crowther, P., et al. (2010). NGC $300 \mathrm{X}-1$ is a WolfRayet/black hole binary, MNRAS. 403, L41-L45.

[14] Romani, R., (1998). A census of low mass black hole binaries, Astron. Astrophys. 333, 583-590.

[15] Liu, J., et al. (2019). A wide star-black-hole binary system from radial-velocity measurements, Nature, 575, 620.

[16] Dias, W., Lepine, J., (2005). Direct Determination of the Spiral Pattern Rotation Speed of the Galaxy, ApJ. 629, 825-832.

[17] Seigar, M., James, P., (1998). The structure of spiral galaxies-II. Near-infrared properties of spiral arms", MNRAS. 299, 685-698.

[18] Conselice C., Wilkinson A., Duncan K., Mortlock, A., (2016). The Evolution of Galaxy Number Density at $\mathrm{z}<8$ and Its Implications, ApJ, 830, 83.

[19] Buta, R., (1995) The catalog of southern ringed galaxies, ApJS. 96, 39-116.

[20] Buta, R., (2017) Galactic rings revisited. II. Dark gaps and the locations of resonances in early-to-intermediate-type disc galaxies, MNRAS. 470, 3819-3849.

[21] Sellwood, J., Carlberg, R., (2019). Spiral instabilities: mechanism for recurrence, MNRAS. 489, 116-131.

[22] Abuter, R., et. al., (2020). Detection of the Schwarzschild precession in the orbit of the star S2 near the Galactic centre massive black hole, A\&A 636, L5 https://doi.org/10.1051/0004-6361/202037813.

[23] Peterken, T., et. al., (2019). A direct test of density wave theory in a grand-design spiral galaxy, Nature Astronomy, 3, 178-182.

[24] Hawking, S., (1998). A brief history of time, (Bantam Books, NY, NY), pp 10. ISBN 0-553-10953-7. 